\title{
The Gravitational Radiation degrees of freedom in Hyperbolic Systems for Numerical Relativity
}

\author{
C. Bona, C. Palenzuela \\ Departament de Fisica. Universitat de les Illes Balears. Ctra de Valldemossa, km 7.5. 07071 Palma de Mallorca. Spain.
}

The gravitational radiation degrees of freedom of freedom are described in the framework of the $3+1$ decomposition of spacetime. The relationship with eigenfields of the KidderScheel-Teukolsky (KST) equations [1] is established. This relationship is used to fix a parameter in the KST equations which is related to the ordering ambiguity of space derivatives in the Ricci tensor, which is inherent to first order evolution systems, like the ones currently used in Numerical Relativity applications.

\section{INTRODUCTION}

The structure of Einstein field equations has deserved great interest since the very beginning of General Relativity. It was early noticed that, by rearranging the order or partial derivatives, the principal part of the four dimensional Ricci tensor could be written as a sort of generalized wave equation [2,3]

$$
2 R_{\mu \nu}=-\square g_{\mu \nu}+\partial_{\mu} \Gamma_{\nu}+\partial_{\nu} \Gamma_{\mu}+\ldots
$$

where the box stands for the d'Alembert operator acting on functions and we have written for short

$$
\Gamma^{\mu} \equiv g^{\rho \sigma} \Gamma_{\rho \sigma}^{\mu}=-\square x^{\mu} .
$$

This opened the way to the use of harmonic spacetime coordinates $\left(\square x^{\mu}=0\right)$ in order to obtain an hyperbolic evolution system [4].

By the middle of the past century, however, the interest focused in the relativistic Cauchy problem. The $3+1$ decomposition of the line element:

$$
\begin{aligned}
d s^{2} & =-\alpha^{2} d t^{2} \\
& +\gamma_{i j}\left(d x^{i}+\beta^{i} d t\right)\left(d x^{j}+\beta^{j} d t\right) \quad i, j=1,2,3
\end{aligned}
$$

allowed one to express six of the ten second order original equations as a system of evolution equations for the metric $\gamma_{i j}$ and the extrinsic curvature $K_{i j}$ of the $t=$ constant slices, namely:

$$
\begin{aligned}
\left(\partial_{t}-\mathcal{L}_{\beta}\right) \gamma_{i j} & =-2 \alpha K_{i j} \\
\left(\partial_{t}-\mathcal{L}_{\beta}\right) K_{i j} & =-\nabla_{i} \alpha_{j}+\alpha\left[{ }^{(3)} R_{i j}-2 K_{i j}^{2}+\operatorname{tr} K K_{i j}\right]
\end{aligned}
$$

where $\mathcal{L}$ stands for the Lie derivative (we restrict ourselves to the vacuum case for simplicity). The remaining four equations could instead be expressed as constraints:

$$
\begin{aligned}
{ }^{(3)} R-\operatorname{tr}\left(K^{2}\right)+(\operatorname{tr} K)^{2} & =0 \\
\nabla_{k} K^{k}{ }_{i}-\partial_{i}(\operatorname{tr} K) & =0 .
\end{aligned}
$$

This opened the door to a new way of obtaining hyperbolic evolution systems [7] 9]. The key point was to use in one or another way the momentum constraint (7) to ensure hyperbolicity while keeping the freedom of choosing arbitrary space coordinates on every $t=$ constant slice. This allows for instance to use normal coordinates $\left(\beta^{i}=0\right)$ without affecting the mathematical structure of the evolution system.(See Ref. 10] for a detailed comparison of the "old" and "new" hyperbolic systems).

These findings came at the right moment for people working on Numerical Relativity with a view on the gravitational waves detector projects starting by the turn of the century. Following the wake of these first works, many groups found their own way of combining the momentum constraint with the evolution equation (5), leading in each case to a new brand of hyperbolic systems [1, 11,25 .

Suddenly, the problem of having too few hyperbolic formalisms for Numerical Relativity turned into the opposite problem of having too many of them. Some of the works considered even multiparameter families of hyperbolic systems [1,10,14]. Faced with the problem of choice, we think that the right question at this point is: what are hyperbolic systems for?

There are many answers, of course, but we can get a hint when we realise that hyperbolic systems basically describe propagation phenomena along characteristic lines. This sets up the question of whether we can use these formalisms in the context of gravitational wave propagation. The $3+1$ formalism does not seem at first sight very useful in that context, because it describes spacetime as a foliation of $t=$ constant hypersurfaces. Then, the natural structure associated with gravitational waves in that formalism is not the light cone itself, but the twodimensional surfaces (wavefronts) obtained as the intersection of the light cone with the $t=$ constant hypersurfaces. The geometry of wavefronts will be discussed in Section II. The second difficulty arises from the fact that, because of the constraint equations (6, 7), only two degrees of freedom correspond to gravitational radiation in spite of the complete (strong) hyperbolicity of the formalisms. This point can be easily illustrated by looking at the list of characteristic speeds given in the KST paper [1] namely $\left\{0, \pm 1, \pm c_{1}, \pm c_{2}, \pm c_{3}\right\}$ where $c_{1}, c_{2}$ and $c_{3}$ depend of a list of arbitrary parameters related with the gauge choice $\left(c_{1}\right)$ and with the way in which the constraints are used to enforce hyperbolicity. It is clear that the degrees of freedom propagating with speed $0, \pm c_{1}, \pm c_{2}$ or $\pm c_{3}$ can not describe gravitational radiation, which we know that propagates at light speed 
$(v= \pm 1)$, independently of how we manage to write down our equations. The two remaining degrees of freedom, as expected, are related with transverse traceless components of the extrinsic curvature $K_{i j}$. We will analyse more closely these eigenfields in Section III.

\section{LIGHT CONES AND WAVEFRONTS}

Let us consider the propagation of a burst of gravitational waves. From the geometrical point of view, it can be described as a foliation of spacetime by a set of null hypersurfaces (light cones), namely

$$
\begin{aligned}
\phi(x, t) & =\text { constant } \\
d \phi \cdot d \phi & =0
\end{aligned}
$$

From every $t=$ constant hypersurface, the propagation is seen instead as a succession of wavefronts. It can be obtained by setting $t=t_{o}$ in (8) to obtain

$$
\phi\left(x, t_{o}\right)=\text { constant }
$$

so that the null foliation ( 8 ) induces a spacelike foliation (10) by two-dimensional surfaces (wavefronts) on every $t=$ constant slice.

Let us consider now an adapted coordinate system on the $t=t_{o}$ slice so that the space unit normal $n_{k}$ to the wavefront $(10)$ is given by

$$
n_{k}=N \delta_{k}^{z}
$$

and the other two coordinates $x^{a}(a=1,2)$ display the wavefronts surface. The line element for the $t=t_{o}$ slice can then be written as $(2+1$ decomposition)

$$
\begin{aligned}
& \gamma_{i j} d x^{i} d x^{j}=N^{2} d z^{2} \\
& \quad+\sigma_{a b}\left(d x^{a}+\lambda^{a} d z\right)\left(d x^{b}+\lambda^{b} d z\right) \quad a, b=1,2
\end{aligned}
$$

where $\sigma_{a b}$ is the induced metric on every wavefront surface, so that it describes the intrinsic geometry of the wavefronts.

The extrinsic geometry of the wavefront foliation (10) is in turn described by the second fundamental form $\kappa_{a b}$, namely

$$
\kappa_{a b}=\frac{1}{2 N}\left(\partial_{z}-\mathcal{L}_{\lambda}\right) \sigma_{a b} .
$$

Both $\sigma_{a b}$ and $\kappa_{a b}$ are $2+1$ tensors, in the sense that they transform in a covariant way under changes of coordinates of the form

$$
\begin{aligned}
\tilde{z} & =f(z) \\
\tilde{x}^{a} & =h^{a}(x, z)
\end{aligned}
$$

which transform the wavefront foliation (10) into itself.

Notice that $\sigma_{a b}, \kappa_{a b}$ can be easily expressed in terms of the three-dimensional metric $\gamma_{i j}$ and the spacelike unit normal $n_{k}$, namely

$$
\begin{aligned}
\sigma_{a b} & =\gamma_{a b} \\
\kappa_{a b} & =-n_{k} \Gamma_{a b}^{k}
\end{aligned}
$$

where $\Gamma^{k}{ }_{i j}$ stand for the Christoffel symbols of $\gamma_{i j}$.

\section{SOLVING THE ORDERING AMBIGUITY}

Let us come back now to the results of the KST paper [1]. The principal part of the first order evolution systems presented there can be written in Flux-Conservative form for the array of variables $u=\left\{\gamma_{i j}, K_{i j}, d_{k i j}\right\}$, namely

$$
\frac{1}{\alpha} \partial_{t} u+\partial_{k} F^{k}(u)=\ldots
$$

so that propagation along the direction given by $n_{k}$ can be studied by solving the following characteristic eigenvalue problem

$$
F^{n}(u) \equiv n_{k} F^{k}(u)=v u
$$

From the transverse components $K_{a b}$, one has [1]

$$
\begin{aligned}
F^{n}\left(K_{a b}\right) & =\frac{1}{2}\left[d_{a b}^{n}-(1+\zeta) d_{(a b)}^{n}\right. \\
& \left.+m \gamma_{a b}\left(d_{k}^{n k}-d_{k}^{k n}\right)\right]
\end{aligned}
$$

so that the parameter $m$ (corresponding to $\gamma$ in [1]) does not contribute to the transverse traceless part. On the other hand, one has [1]

$$
\begin{aligned}
& F^{n}\left(d_{n a b}\right)=2 K_{a b}-\chi \gamma_{a b}\left(K^{n n}-t r K\right) \\
& F^{n}\left(d_{a b n}\right)=-\frac{\eta}{2} \gamma_{a b}\left(K^{n n}-t r K\right)
\end{aligned}
$$

so that it is clear again that the parameters $\eta, \chi$ do not contribute to the transverse traceless part.

This means that the traceless part of the combinations

$$
K_{a b} \pm \frac{1}{2}\left[d_{a b}^{n}-(1+\zeta) d_{(a b)}^{n}\right]
$$

corresponds to eigenfields propagating with light speed $(v= \pm 1$, respectively). The parameter $\zeta$ appearing in (23) is related with the ordering ambiguity inherent to first order formalisms, where the space derivatives $d_{k i j}$ are considered to be independent quantities, so that the identity

$$
\partial_{[r} d_{s] i j}=0
$$

is not granted and must be considered as a supplementary constraint [1]. The value $\zeta=-1$ corresponds to a decomposition of the three-dimensional Ricci tensor of the form (1), which is closer to the wave equation. The value $\zeta=+1$ corresponds instead to the simpler decomposition

$$
{ }^{(3)} R_{i j}=\partial_{k} \Gamma^{k}{ }_{i j}-\partial_{(i} \Gamma^{k}{ }_{j) k}+\Gamma_{k r}^{k} \Gamma^{r}{ }_{i j}-\Gamma_{r i}^{k} \Gamma_{k j}^{r}
$$

Notice that, allowing for (17), the combinations (23) in the $\zeta=+1$ case can be written as

$$
K_{a b} \pm \kappa_{a b}
$$

so that they have a geometrical meaning in terms of the extrinsic curvature of the $t=$ constant hypersurfaces and 
the wavefront foliation. For any other value of $\zeta$, however, the eigenfields described by the traceless part of (23) are not covariant under the coordinate transformations (14) and do not admit then any consistent, coordinate independent, physical interpretation. It follows that one can solve the ordering ambiguity for the derivatives in the principal part of the Ricci tensor by requiring that the eigenfields corresponding to the transverse traceless degrees of freedom do have a clear geometrical meaning in terms of the geometry of the $t=$ constant hypersurfaces and that of the wavefronts. This requirement amounts to select the particular value $\zeta=+1$, corresponding to the classical decomposition (25).

\section{CONCLUDING REMARKS}

The parameter dependence of the eigenfields (23) may seem surprising at the first sight, because these transverse traceless degrees of freedom are related with a real physical phenomenon (gravitational waves), which is independent on how we do actually decompose the Ricci tensor. This paradox can be solved by noticing that the Fluxes appear in the evolution equations (18) under a divergence operator; the parameter $\zeta$ appears when including the "rotational" constraint (24), but all these terms just cancel under the divergence operator, so that all values of $\zeta$ are physically equivalent.

The special choice $\zeta=+1$ corresponds to the only case in which the Fluxes themselves inherit the covariance properties of the equations. We claim that this is an important feature, because then gravitational radiation features are explicitly inherited by the eigenfields of the system.

On the other hand, there are indications that $\zeta=+1$ may not be convenient from the Numerical Relativity point of view. Most of the formalisms that are being currently (and successfully) used to that end actually imply the opposite choice $(\zeta=-1)$. These include at least the Bona-Massó [8,12], Shibata-Nakamura [26], BaumgarteShapiro [27] and Anderson-York [25] formalisms. The reason may be that with the $\zeta=-1$ choice the transverse traceless part of the evolution system looks like that of the wave equation, as pointed out in [1], which is known to admit a symmetric-hyperbolic form.

Symmetric hyperbolicity is a stronger requirement than just strong hyperbolicity in order to ensure existence, unicity and stability of the solutions arising from a given set of initial data (well posedness) [28]. When the evolution system is just strongly hyperbolic, but not symmetric hyperbolic, then well posedness is not ensured for non-smooth data, like the ones arising in numerical experiments due to finite machine accuracy.

We actually have some preliminary evidence that the choice $\zeta=+1$ is prone to numerical instabilities. To confirm this, one must proceed to a systematic exploration of parameter space like the one presented in the KST paper
[1] for the $\zeta=-1$ case. There is also some theoretical evidence [29] that the choice $\zeta=+1$, when combined with conventional choices of the other parameters introduced in [1], does not lead to a symmetric hyperbolic system. This opens the way, from the theoretical point of view, to using the symmetric hyperbolicity requirement, in the $\zeta=+1$ case, to restrict the values of the other parameters, then reducing the volume of parameter space.

We are currently working along these lines, both from the theoretical and from the numerical point of view.

Acknowledgements: We thank Manuel Tiglio for helpful and stimulating discussions during his visit to Palma de Mallorca. This work has been supported by the EU Programme 'Improving the Human Research Potential and the Socio-Economic Knowledge Base' (Research Training Network Contract (HPRN-CT-2000-00137), by the Spanish Ministerio de Ciencia y Tecnologia through the research grant number BFM2001-0988 and by a grant from the Conselleria d'Innovacio i Energia of the Govern de les Illes Balears.

[1] L. E. Kidder, M. A. Scheel and S. A. Teukolsky, Phys. Rev. D64, 064017 (2001).

[2] T. De. Donder,La Gravifique Einstenienne GauthierVillars, Paris (1921).

[3] C. Lanczos, Phys. Z. 23537 (1922).

[4] Y. Choquet-Bruhat, Acta Math. 88141 (1955).

[5] S. W. Hawking and G. F. R. Ellis, The large scale structure of spacetime, Cambridge U.P. (1973).

[6] D. DeTurck, Invent. Math. 65179 (1981).

[7] Y. Choquet-Bruhat and T. Ruggeri, Comm. Math. Phys. 89269 (1983).

[8] C. Bona and J. Massó, Phys. Rev. Lett. 681097 (1992)

[9] S. Frittelli and O. A. Reula, Commun. Math. Phys. 166 221 (1994).

[10] C. Bona in Hyperbolic problems: theory, numerics, applications, Int. Series of Numerical Mathematics, Vol.129, Birkhuser (1999).

[11] A. Abrahams, A. Anderson, Y. Choquet-Bruhat and J. W. York, Phys. Rev. Lett. 753377 (1995).

[12] C. Bona, J. Massó, E. Seidel and J. Stela, Phys. Rev. Lett. 75 600 (1995).

[13] C. Bona, J. Massó, E. Seidel and J. Stela, Phys. Rev. D56 3405 (1997).

[14] S. Frittelli and O. A. Reula, Phys. Rev. Lett. 764667 (1996).

[15] H. Friedrich, Class. Quantum. Grav. 141451 (1996).

[16] M. H. P. M. van Putten and D. M. Eardley, Phys. Rev. D53 3056 (1996).

[17] F. B. Estabrook, R. S. Robinson and H. D. Wahlquist, Class. Quantum. Grav. 141237 (1997). 
[18] M. S. Iriondo, E. O. Leguizamon and O. A. Reula, Phys. Rev. Lett. 794732 (1997).

[19] M. A. G. Bonilla, Class. Quantum. Grav. 152001 (1998).

[20] J. M. Stewart, Class. Quantum. Grav. 152865 (1998).

[21] G. Yoneda and H. Shinkai, Phys. Rev. Lett. 82263 (1999).

[22] H. Shinkai and G. Yoneda, Class. Quantum. Grav. 17 4799 (2000).

[23] G. Yoneda and H. Shinkai, Class. Quantum. Grav. 18 441 (2001).

[24] M. Alcubierre, B. Bruegmann, M. Miller and W. M. Suen, Phys. Rev. D60, 064017 (1999).

[25] A. Anderson and J. W. York, Jr., Phys. Rev. Lett. 82 4384 (1999).

[26] M. Shibata and T. Nakamura, Phys. Rev. D52 5428 (1995).

[27] T. W. Baumgarte and S. L. Shapiro, Phys. Rev. D59 024007 (1999).

[28] H. O. Kreiss and J. Lorentz, Initial boundary value problems and the Navier-Stokes equations, Academic Press, New York (1989).

[29] L. Lindblom in Stability of the KST equations, Talk at the Workshop on Formalisms of Einsteins Equations for Numerical Relativity, Mexico City-UNAM, May 13-24 2002. 